\title{
Counting and Measuring Ultrastructural Features of Biological Samples
}

\author{
Mark J. West
}

\begin{abstract}
Ultrastructural features of cells can be fractions of a micrometer in diameter, and electron microscopy is needed to resolve them to a degree that is compatible with stereological techniques. Because the focal depth of transmission electron microscopy (TEM) images is thousands of times greater than the thickness of the sections used with TEM, virtual sectioning of sections suitable for TEM is not possible, as it is with light microscopy and the optical disector probe. With features the size of neuronal synapses, for example, this necessitates the use of physical sections and physical disectors. Regardless of how the imaging is performed, the design of stereological studies for quantifying ultrastructural features will be essentially the same as that used in the example described here, which uses physically separated ultrathin sections viewed with conventional TEM to estimate the number and size of synapses in a particular brain region.
\end{abstract}

\section{EXAMPLE INVOLVING ESTIMATION OF NUMBER AND SIZE OF SYNAPSES}

The stereological method described here for estimating the total number of synaptic contacts $N_{\text {SYN }}$ in a well-defined region of the brain is based on a two-step procedure. This approach has been shown to be applicable to regions of the nervous system that vary significantly in absolute volume (Geinisman et al. 1996; Scheff et al. 2007; West et al. 2009). The total number of synapses $N_{\mathrm{SYN}}$ can be estimated as the product of an estimate of the volume of the region of interest or reference volume $V_{\mathrm{REF}}$ made by point counting and an estimate of the numerical density of synapses made $N_{\mathrm{V}_{\mathrm{SYN}}}$ with physical disectors:

$$
N_{\mathrm{SYN}}=V_{\mathrm{REF}} \times N_{\mathrm{V}_{\mathrm{SYN}}} \text {. }
$$

Standard fixation and staining methods used in conventional electron microscopy of the nervous system can be used for the stereological analysis of synapses.

In the example presented here, $V_{\mathrm{REF}}$ is the stratum radiatum of $\mathrm{CA} 1$ of the hippocampus, that is, srCA1, of the C57Bl/6 mouse. The tissue was prepared according to traditional electron micrograph (EM) protocols. Subsequently, lead citrate was used to stain ultrathin sections of tissue after embedding in Epon. This tissue proved to be suitable for the identification of synaptic contact specializations. Selective staining of the paramembranous specializations with ethanolic phosphotungstic acid (EPTA) can be used under special circumstances and with some caveats. EPTA has been used in human postmortem material to stain synaptic contacts (Tang et al. 2001), which are apparently more

Adapted from Basic Stereology for Biologists and Neuroscientists by Mark J. West. CSHL Press, Cold Spring Harbor, NY, USA, 2012. 
M.J. West

robust than the membranes of cells and organelles. However, the lack of membrane features in these preparations makes it difficult to differentiate between segmented and nonsegmented postsynaptic densities (PSDs), and between synaptic contacts and other cellular organelles such as mitochondria, spine apparati, and lysosomes that stain in EPTA-stained tissue.

\section{SAMPLING THE TISSUE}

Estimating $V_{\text {REF }}$

\section{Preparing a Systematic Series of Sections for Estimating $V_{R E F}$}

The first phase of the sampling involves preparing the material so that an unbiased estimate can be made of a brain region from a series of parallel sections through the entire region of interest, $V_{\text {REFF }}$. As pointed out in Estimating Volume in Biological Structures (West 2012a), an unbiased estimate of the volume of a region of interest can be obtained using point-counting techniques and Cavalieri's principle. Regardless of the size of the region of interest (the hippocampus of an elephant or a mouse), 12-18 equally spaced sections will be needed to make the estimate of the reference volume. (The rationale for considering this number of sections to be optimal is described in The Precision of Estimates in Stereological Analyses [West 2012b] and Optimizing the Sampling Scheme for a Stereological Study: How Many Individuals, Sections and Probes Should Be Used [West 2013a].) In the case of the example provided here, for the mouse srCA1, this was accomplished by cutting the entire brain into $300-\mu \mathrm{m}$-thick slabs with a vibratome, staining and embedding the portions of the slabs that contained hippocampus, and cutting one semithin 1- to 2 - $\mu$ m-thick section from the corresponding surfaces of each of the slabs. This resulted in 12-14 equally spaced sections throughout the entire region of interest. The maximum thickness of the slabs should not exceed $1 \mathrm{~mm}$ to ensure that the tissue can be thoroughly osmicated, block stained, and infiltrated with embedding medium. Figure 1 is a depiction of a series of parallel sections through the hippocampus from one side of a mouse brain that were used to estimate the volume of srCA1, $V_{\text {srCA1 }}$.

As pointed out in The Precision of Estimates in Stereological Analyses (West 2012b), Systematic versus Random Sampling in Stereological Studies (West 2012c), Getting Started in Stereology (West 2013b), and Optimizing the Sampling Scheme for a Stereological Study: How Many Individuals, Sections and Probes Should Be Used (West 2013a), one usually does not need more than 150 points in all to estimate the volume of regions with complex shapes, if one has between 12 and 18 sections. However, in this example, a very large number of points was used to facilitate the process of defining the positions to be sampled for $N_{\mathrm{V}}$, as described in the section Relationship Equations for $\mathrm{S}$ and $\mathrm{L}$ are Tissue Orientation Sensitive in Isotropy, iSectors, and Vertical Sections in Stereology (West 2013c). This is relatively easy to achieve with the "autofill" feature of stereology software packages.

$$
V_{\text {srCAl }}=\Sigma P_{\text {srCA1 }} \times A_{\mathrm{P}} \times T
$$

\section{Delineation of Region of Interest is Critical}

Delineation of the region of interest is critical for the estimation of the total number of synapses and is the most likely source of discrepancies in stereological studies performed by different investigators. The statistical significance of differences in total number of synapses in any one study can also be affected by small variations in the delineation process. It is therefore important that rigorous definitions of the borders be established a priori and reported in detail. In the example used here, srCA1, the borders with the subiculum and molecular layer are particularly subject to investigator variability (Fig. 2) (West et al. 2009). It is also important that the preparation and analysis of the tissue be performed without investigator knowledge of the group identity of the mice, so that any variability in the delineation process contributes equally to the variances of estimates used for comparative purposes. 

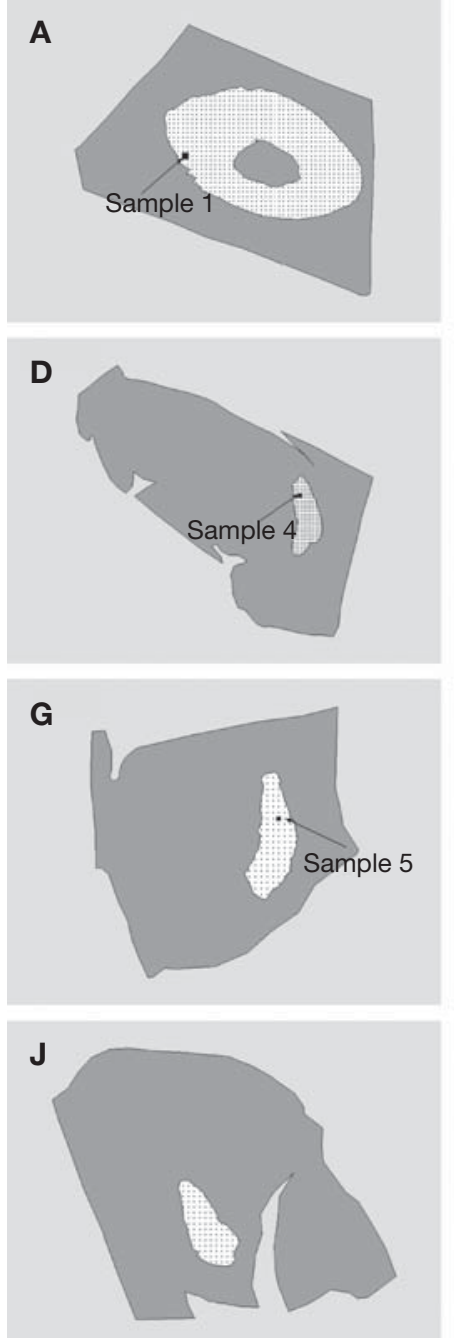
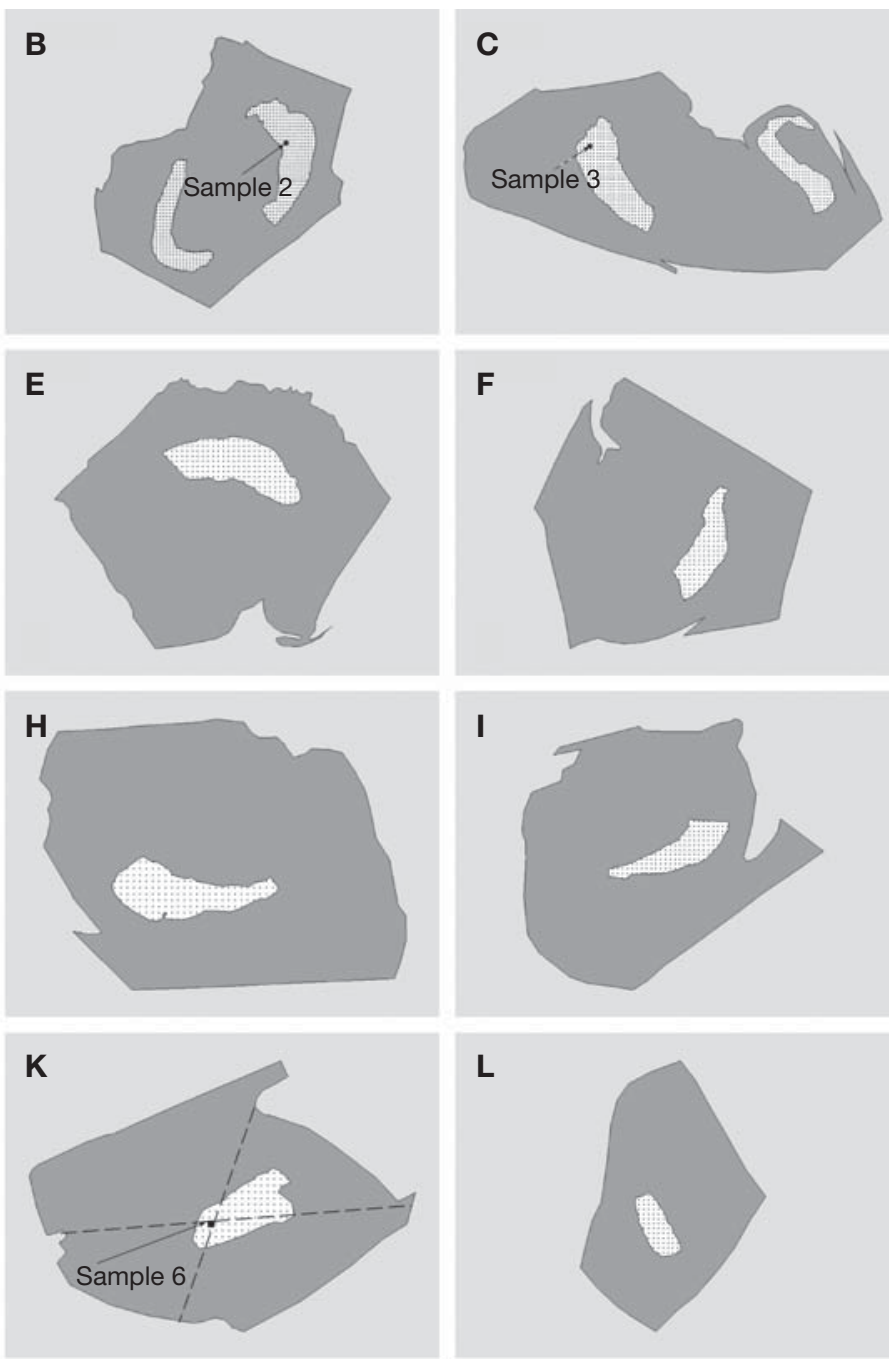

FIGURE 1. Graphic display of tracings of 1 - $\mu$ m-thick sections from the faces of the slabs (blocks) from one mouse showing the outlines of the embedded tissue slabs and $\operatorname{srCA} 1(A-L)$. The latter were filled with points, with a known area associated with each point $\left(A_{p}=2500 \mu \mathrm{m}^{2}\right.$ in this example). These, in turn, were used to estimate the volume of the stratum radiatum (srCA1) and, notably, also to define the six positions to be sampled for electron microscopic analysis (samples $1-6$, shown in $A, B, C, D, G$, and $K$ ). The different sections depicted in the figure are presented at different magnifications to maximize the outlines of the embedded slabs of hippocampal tissue. Features of the outlines of the embedded tissue in the sections were used to triangulate the positions of the tissue on the block face that were ultimately serial sectioned, as shown in K. (Redrawn from West et al. 2009.)

\section{Estimating $N_{V}$}

\section{Specifying Positions to be Sampled for Numerical Density}

To estimate numerical density $N_{\mathrm{V}}$ of synapses, the positions within the region of interest that are to be sampled with disectors must be chosen so that all parts of the region of interest, srCA1, have equal probabilities of being sampled.

In the example given here for srCA1, the sections used to estimate the reference volume (see Fig. 1) can be used to specify these positions. These sections have been chosen in a systematic random manner along one axis of the tissue, that is, the first section has a random placement within an interval specified by the slab thickness. This is because one initiates the vibratoming without knowledge of where the first section will be placed in the region of interest, for example, the hippocampus, and as a consequence, subsequent sections are positioned at uniform intervals thereafter and will 


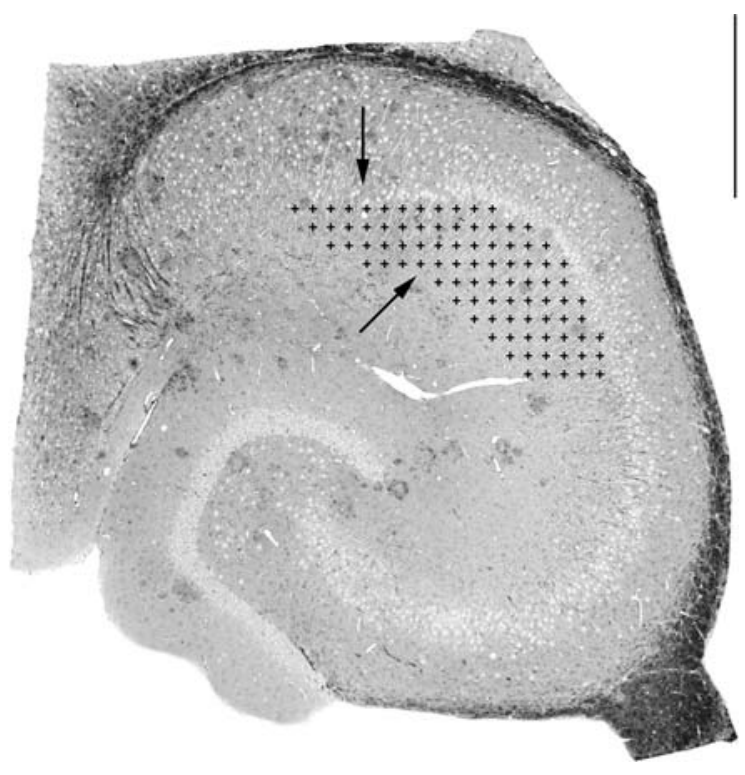

FIGURE 2. Semithin transverse Epon section through the hippocampus of a transgenic mouse stained with Toluidine Blue showing srCA1 marked by a black point-counting grid. Critical borders are indicated by arrows. Scale bar, $500 \mu \mathrm{m}$. (Redrawn from West et al. 2009.)

constitute a systematic random series of sections. Sections chosen in this manner have an equal probability of containing any synapse in srCA1. Because of the very large number of synapses that can be expected to be in any one slab, it is necessary to limit the next step in the sampling, that is, with disectors, to small regions of the slabs. These subsamples must be positioned so that all parts of the surface of the slabs (all synapses at that level) have equal probability of being sampled. They are positioned at a systematic random set of positions within the $x, y$ dimensions of the surface of the slabs. As a consequence, the amount of sampling performed on any one slab is proportional to the area of the cut surface of the slab. This can be done with the aid of the point-counting grid used to estimate $V_{\mathrm{REF}}$, as shown in Figure 1.

In the example presented here for srCA1, six systematic random positions within the three dimensions of the region of interest were sampled to obtain estimates of $N_{\mathrm{V}}$. A more detailed discussion follows regarding the amount of positions as well as the reasons why six positions should be sampled in this example. First, a method for physically specifying the actual positions of any number of samples is considered. The sum of the areas of the profiles of the region of interest on the semithin sections used to estimate $V_{\mathrm{REF}}$ (see above) corresponds to the total area of the sections to be sampled. Dividing this area by the number of samples one wishes to position within the three dimensions (in this example, six) results in an area that corresponds to the distance, in two dimensions, between the positions to be sampled on the sections. A similar process is described for the spacing of 150 optical disector samples in Getting Started in Stereology (West 2013b). (For more information on optical disectors, see the section entitled The Optical Disector Probe in Estimating Object Number in Biological Structures [West 2012d].) Because each point used in the point-counting grid has a known area associated with it (Fig. 1), the area of the profile of the region of interest, srCA1, can also be expressed by the number of points that will hit the profile. The area between samples is equivalent to a set number of points. After a random start within the first interval of this number of points, the points at intervals of this number can be defined subsequently (Table 1). The positions of these points can be viewed on images of the semithin sections used for point counting (Fig. 1). Graphic representations of the superimposed tracings of the outlines of the edges of the slab of embedded tissue, boundaries of the region of interest, and representations of the point-counting grid can then be used to identify the positions in the sections to be sampled. Using features at the edge of the slab and the position of the sample (a point), corresponding positions on the surface of the block face can be defined, by triangulation, and small regions of the block face at those positions trimmed down for serial ultramicrotomy. 
TABLE 1. Portion of a worksheet that contains point-count data from the sections shown in Figure 3

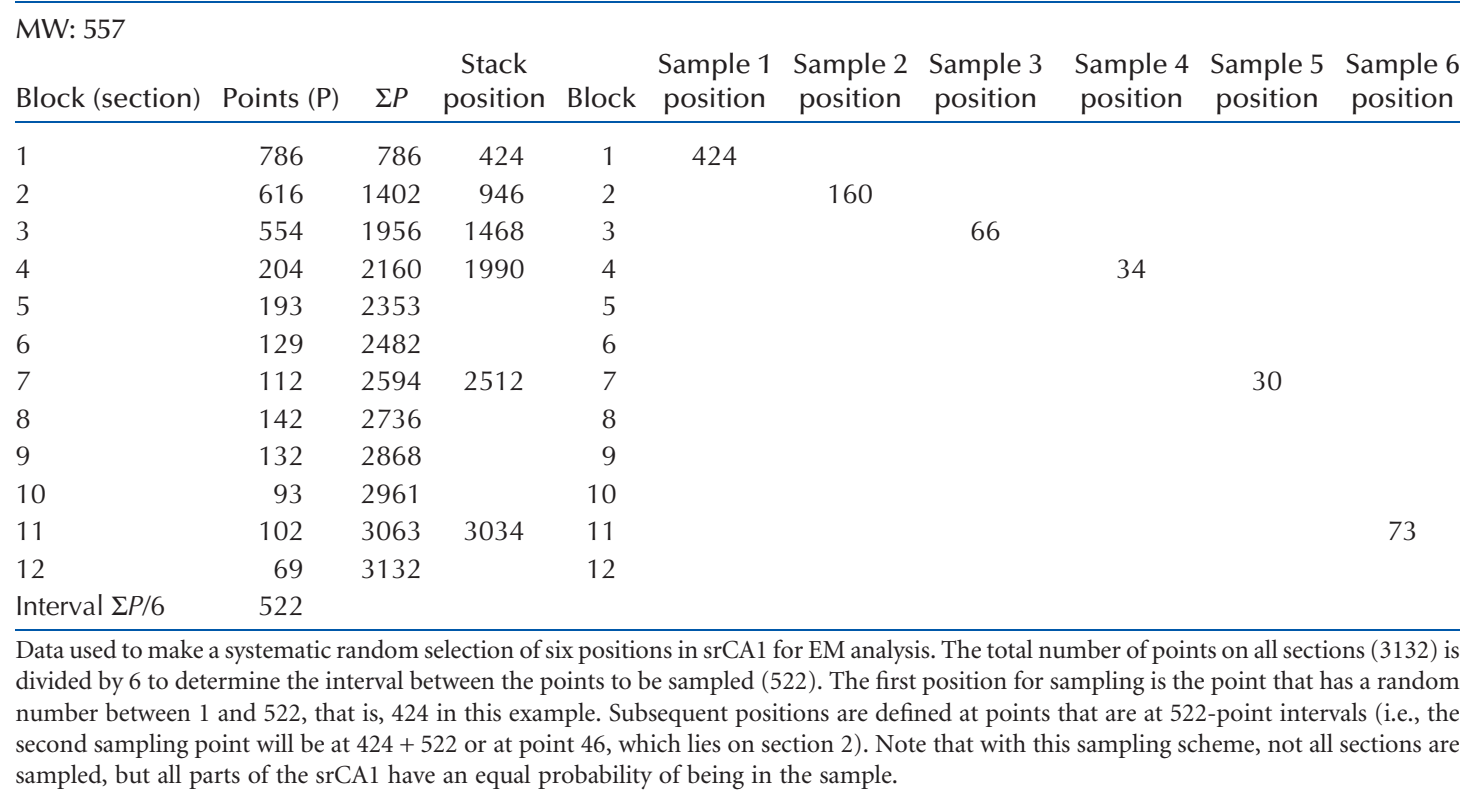

\section{Number of Stacks Needed}

Returning to the issue of how many sites should be sampled with physical disectors to obtain an estimate of $N_{V}$, it should be pointed out once again that there is no set a priori rule. In general, the answer can only be derived after a pilot analysis of the sources of variance that contribute to the parameter that is ultimately used in the comparisons, for example, of group means. (For more details regarding this process, see Optimizing the Sampling Scheme for a Stereological Study: How Many Individuals, Sections and Probes Should Be Used [West 2013a].) The primary factor affecting the variability in the samples of $N_{\mathrm{V}}$ is the homogeneity of the distribution of synapses in the region of interest. If the numerical density is the same in all parts of the region of interest, one sample of 100200 neurons should suffice. However, because we do not want to assume anything regarding organization of the structures, such as the absence of gradients in the numerical density of synapses along the three axes of the region of interest, at least two (random) samples are required to develop some feeling for the variability in the estimate of $N_{\mathrm{V}}$. In an earlier study of synapse number based on similar principles (Geinisman et al. 1996), six samples were shown to capture enough of the variability in numerical density that the variance of the final estimator of total number (which is the sum of the variances of the estimates $N_{\mathrm{V}}$ and $V_{\mathrm{REF}}$ ) was sufficient for comparative studies (Geinisman et al. 2004). In the original study, the use of six samples was considered to be a reasonable balance between variability in synapse density that might be expected from site to site and the amount of work necessary to make each individual sample of numerical density at a site, that is, cut, image, and analyze a stack of physical densities. Again, the appropriateness of using six samples can only be confirmed after comparisons of parameters at higher levels of the sampling scheme, such as group means. (See discussion of analysis of variance in Optimizing the Sampling Scheme for a Stereological Study: How Many Individuals, Sections and Probes Should Be Used [West 2013a].) In the example used here, this turned out to be the case when six samples were used to estimate the $N_{\mathrm{V}}$ in each individual, in that the mean observed relative variance $\overline{\mathrm{OCE}}^{2}$ of the estimator of $N_{\mathrm{SYN}}$ was less than the observed relative variance of the group mean (see Eq. 1 in The Precision of Estimates in Stereological Analyses [West 2012b]). The estimate of the numerical density in an individual is the mean of a set number of samples within that individual. In the example given here, there are six samples of $N_{\mathrm{V}}$ in each individual. 


\section{Example of Sampling with Stacks}

In the example given here, a serial stack of electron micrographs was collected at each of the six positions to be sampled. The magnification of the micrographs and the number of sections were chosen so that, on average, $\sim 30$ synaptic contacts would be sampled ("counted"). This number ensured that $\sim 150$ synaptic contacts would be sampled when all six samples were collected (see The Precision of Estimates in Stereological Analyses [West 2012b] and Optimizing the Sampling Scheme for a Stereological Study: How Many Individuals, Sections and Probes Should Be Used [West 2013a] for why 150 samples is an appropriate starting point). Magnification of the images should be the lowest at which the objects of interest—in this case, synaptic contacts—can be clearly identified. The lowest magnification at which this can be done was chosen because this results in sampling the largest area of the sections. The larger the area sampled, the larger the volume of tissue contained in a stack of images. The volume of the tissue being sampled is also defined by the number of sections in the stack and the thickness of the sections.

The primary magnification was 13,500×. Gold section series were collected for analysis. After collection on Formvar slot grids, the ultrathin sections were stained with electron-dense metals.

\section{Defining and Counting Synaptic Contacts}

In this example, synaptic contacts were defined as postsynaptic electron-dense membrane specializations or (PSDs that have at least three vesicles in close proximity to associated presynaptic synaptic densities. Without referring to adjacent micrographs, synaptic contacts were interactively marked on the digital images. To facilitate the alignment of the serial electron micrographs, micrographs were confined to the lower-right-hand corner of each of the ultrathin sections in the serial ribbon of sections (Fig. 3).

Consecutive pairs of micrographs in each stack were viewed, and profiles of synaptic contacts belonging to the same synapse were then given a unique synapse identification that was used to determine the number and sizes of synapses in a stack. Proceeding pairwise through the stack of micrographs, that is, $1-2,2-3,3-4$, and so on, profiles of synapses were counted in the second section of the pair, if they were sampled by the superimposed counting frame and did not have a recognizable profile in the first section, $\Sigma Q_{\text {SYN }}^{-}$(Figs. 4 and 5; Table 2), that is, with physical disector counting rules. The volume sampled by each stack of physical disectors $V_{\text {STACK }}$ was calculated as the product of the area of the counting frame $a_{\text {frame }}\left(330 \mu \mathrm{m}^{2}\right)$; the thickness of the sections $t$ (which was estimated to be $100 \mathrm{~nm}$ on the basis of color); and the number of sections in the stack $s$ using Equation 3. A summary of the parameters that define the sampling scheme appears in Table 3.

$$
V_{\text {STACK }}=A_{\text {frame }} \times t \times s .
$$

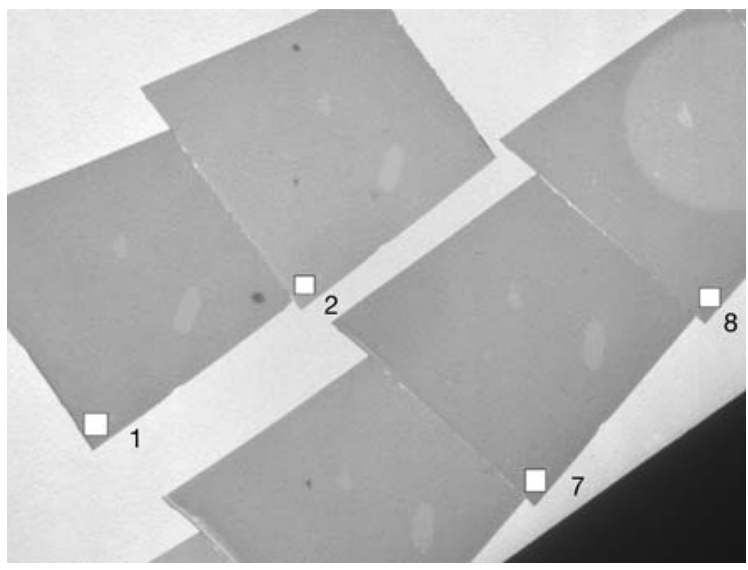

FIGURE 3. Low-power electron micrograph of a series of ultrathin sections mounted on the carbon film of a slot grid. To aid in the alignment of the serial stack of physically separate sections, micrographs were taken of the same corner of the sections (small open squares). 


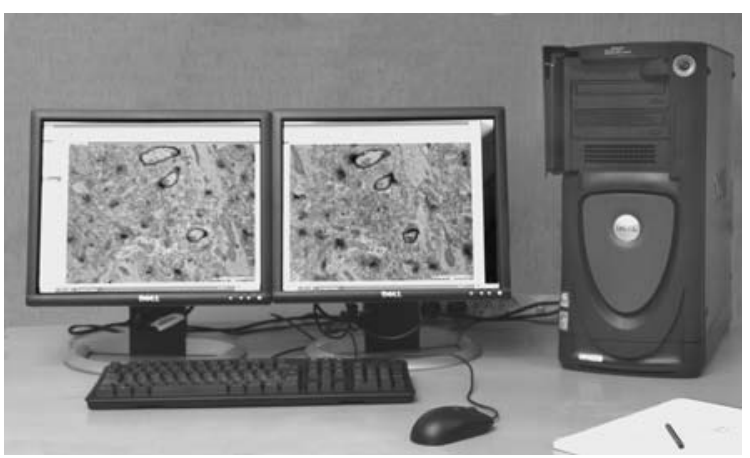

FIGURE 4. Consecutive pairs of sections were analyzed while being viewed simultaneously on two computer monitors.

In Figure 5, all sectional profiles of synaptic contacts are marked (boxes) on the individual digital electron micrographs. Profiles belonging to the same contact are given a unique contact identification number. Contacts that have their first identifiable sectional profile below the first section in the series are counted (Table 2), provided that they are sampled by the superimposed unbiased areal counting frame (thin lines around the edge of the micrograph in Fig. 5) (see Estimating Object Number in Biological Structures [West 2012d]). Volume of tissue sampled is calculated as the product of the area of counting frame $A_{\text {frame }}$, section thickness $t$, and number of sections in stack $s$. The largest length of the profiles that belonged to the same synaptic contact is defined as the width of the synaptic contact (Table 1).

There are some caveats that should be mentioned with regard to this measure of synaptic contact size, because this measure may not be truly unbiased. First, it is assumed that the contact surfaces are randomly oriented; this was not tested. Second, there may be synaptic contacts that are not identifiable in the stack because the synaptic cleft lies perfectly in the plane of the section. It has been estimated that this can produce an underestimation in total number $N_{\mathrm{SYN}}$ by $3 \%$ (Tang et al. 2001).

The numerical density of synaptic contacts in each of the six stacks $N_{\mathrm{V}_{\text {SYN (STACK) }}}$ sampled in an individual was determined by dividing the number of synapses counted in the stack, $\Sigma Q_{\text {SYN }}^{-}$, by the volume of the stack $V_{\text {STACK }}$ as shown in the following equation:

$$
N_{\mathrm{V}_{\mathrm{SYN}(\mathrm{STACK})}}=\frac{\Sigma Q_{\mathrm{SYN}}^{-}}{V_{\mathrm{STACK}}}
$$

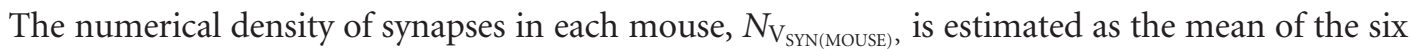
samples of numerical density made in that mouse (Eq. 5). Accordingly, the variance of the estimates of the numerical density made in each mouse $N_{\mathrm{V}_{\text {SYN(MOUSE) }}}$ is calculated on the basis of a sample size $n$ of six

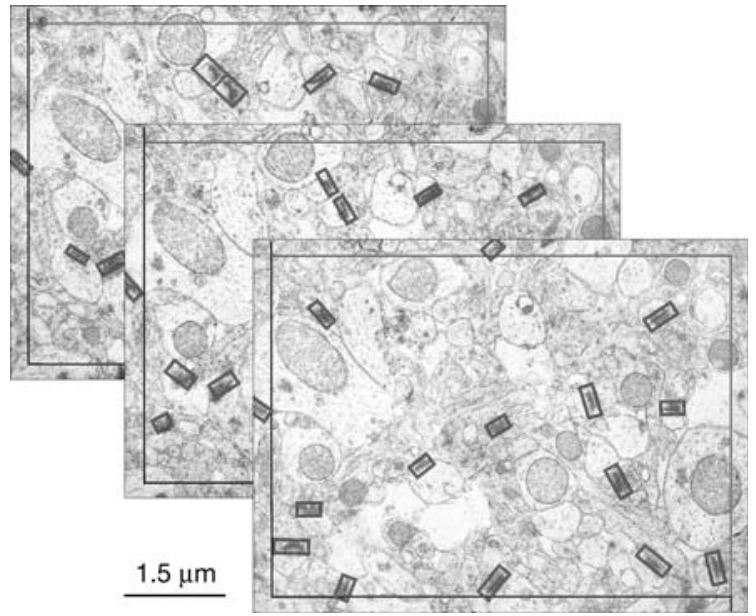

FIGURE 5. Three serial sections from a larger series illustrating counting and measuring procedures performed at each sampling site within srCA1 described in the text. (Redrawn from West et al. 2009.) 
TABLE 2. Stack analysis

\begin{tabular}{|c|c|c|c|c|c|c|c|c|c|c|c|c|}
\hline Synapse section & 1 & 2 & 3 & 4 & 5 & 6 & 7 & 8 & 9 & 10 & 11 & 12 \\
\hline 1 & 309 & 311 & & & & & & & & & & \\
\hline 2 & 236 & & 289 & 312 & & & & & & & & \\
\hline 3 & & & 309 & 514 & & & & & & & & \\
\hline 4 & & & 235 & & 427 & 349 & 545 & 414 & & & & \\
\hline 5 & & & & & & 218 & 447 & 181 & & & & \\
\hline 6 & & & & & & 211 & & 268 & & & & \\
\hline 7 & & & & & & 239 & & & & & & \\
\hline 8 & & & & & & & & & 400 & & & \\
\hline 9 & & & & & & & & & 719 & 234 & & \\
\hline 10 & & & & & & & & & 1069 & 255 & & \\
\hline 11 & & & & & & & & & 794 & 360 & 264 & 329 \\
\hline 12 & & & & & & & & & & & & 301 \\
\hline
\end{tabular}

independent samples (stacks) of the numerical density:

$$
N_{\mathrm{V}_{\mathrm{SYN} \text { (MOUSE) }}}=\operatorname{mean}_{1-6} \frac{\Sigma Q_{\mathrm{SYN}}^{-}}{V_{\mathrm{STACK}}} .
$$

\section{Ultrathin Section Thickness $\mathrm{t}$}

The thickness at which the ultrathin sections in the stack are cut is determined by two factors. The sections must be thin enough to be able to identify the objects, and they should be as thick as possible to maximize the volume of the physical disectors. In the example used here, 100-nm-thick sections were chosen with this strategy in mind. Although sections of this thickness do not have the clarity of the thinner sections often used in descriptive publications, they do contain more information and thereby require fewer sections to obtain a sample of a certain size.

\section{Numbers of Sections in a Serial Stack}

The number of sections in the stack $s$ should be such that the sum of the counts obtained from all of the stacks in an individual should be $\sim 150$. Accordingly, in the example given here, the stack contains $\sim 30$ synaptic contacts, on average (see Getting Started in Stereology (West 2013b) for an explanation as to why counting $\sim 150$ synapses is appropriate at the beginning of a study). Because the magnification has been chosen to be the lowest at which synaptic contacts can be identified, a pilot study

TABLE 3. Summary of sampling scheme for estimating synapse number and size

\begin{tabular}{ll}
\hline$A_{\mathrm{P}}$ used to estimate $V_{\text {SrCA1 }}$ & $2500 \mathrm{~mm}^{2}$ \\
$T$ used to estimate $V_{\text {srCA1 }}$ & $300 \mu \mathrm{m}$ \\
Sections used to estimate $V_{\text {srCA1 }}$ & 12 \\
EM stacks per mouse & 6 \\
$S$ sections per EM stack & $8-14$ \\
$A_{\text {frame, }}$ EM & $3.3 \times 10^{8} \mathrm{~mm}^{2}$ \\
$t, E M$ & $100 \mathrm{~nm}$ \\
$\Sigma Q_{S Y N}^{-}$for estimate of $N_{\text {SYN }}$ & $210(146-295)$ \\
$\Sigma Q_{S Y N}^{-}$for estimate of $A_{\text {SYN }}$ & $181(126-247)$ \\
\hline$\left(V_{\text {srCA1 }}\right)$ Volume of stratum radiatum CA1 of hippocampus, $\left(A_{\mathrm{P}}\right)$ area per point on point grid, $(T)$ \\
distance between corresponding surfaces of slabs used to estimate $V_{\text {srCAl }},(s)$ sections in a stack, $\left(A_{\text {frame }}\right)$ \\
area of disector counting frame, $(t)$ section thickness, $\left(\Sigma Q_{\text {SYN }}^{-}\right)$sum of synapses counted in six stacks.
\end{tabular}


indicated that stacks that were on the order of $\sim 10$ sections would be adequate to achieve this when sections are $100 \mathrm{~nm}$ thick. Although disector stacks with these dimensions can be expected to be applicable to homologous brain regions in other species, analyses of other brain regions, organelles, and organs require similar pilot studies.

\section{Distance between Sections in the Disector Stack}

When sampling structures such as synaptic contacts, which can have diameters $<200 \mathrm{~nm}$, it is prudent to record images of immediately adjacent serial sections when generating the stack, lest the small synapses go undetected. Theoretically, one can use any distance between the two sections of a disector, provided that the distance is not so great that objects "disappear" between sections. In general, this can be ensured if the distance between the sections is half of the smallest dimension of the objects. In the example presented here, every section was used in the stack to avoid missing the smallest synaptic contacts.

\section{Measuring Section Thickness t}

To make an estimate of the numerical density, it is necessary to know the volume of the tissue in which the objects-synaptic contacts—are counted. The volume of each disector stack is the product of the area of the section being sampled $A_{\text {frame, }}$ which one can measure on the micrograph when the magnification is known, and the thickness of the stack, which is the number of sections $s$ in the stack, times the average thickness of the sections in the stack $\bar{t}$ (Eq. 3). To determine the volume of the disector stack, one must have information regarding the thickness of the ultrathin sections in the stack. There are several ways to obtain this information, some easier than others. The more rigorous methods include those based on measurements of cylindrical structures in the tissue (Fiala and Harris 2001a) and Small's fold method based on measuring the width of folds in the section (DeGroot 1988). In some cases, it may not be possible to consistently identify the folds or cylindrical structures needed to use these methods.

In the example given here, only series of gold sections were collected for analysis. Sections of this color may range in thickness from 85 to $115 \mathrm{~nm}$ (Hayat 1979). As pointed out above, this relatively large section thickness was chosen to maximize the volume of physical disectors and maintain enough morphological detail for proper identification of synaptic contacts. Any systematic deviation in the section thickness from the 100-nm thickness used in the example can be expected to introduce a deviation in the estimates of total synapse number from the true value by a factor that is directly proportional to the deviation, that is, $15 \%$. This potential bias may account for the slight differences in synapse numerical density in the example provided here as well as those of other investigators, as discussed by West et al. (2009). A technique for counting synapses based on fractionator sampling that eliminates the need for measuring the thickness of ultrathin sections has been described (Witgen et al. 2006).

It is important to point out that if the investigators are blind to the identity of the material during all phases of preparation and analysis and if the tissue is processed and analyzed in random order, variations in section thickness can be expected to be equally distributed in the material and will add to the variance but not the magnitude of any group differences.

\section{ATROPHY AND TISSUE DEFORMATION}

In the example provided here, the calculations used to estimate the total number of synapses take brain atrophy, postmortem shrinkage, and any shrinkage related to tissue preparation into account. The density of synapses $N_{\mathrm{V}_{\mathrm{SYN}}}$ - that is, the number per unit volume of tissue estimated with physical disectors - is multiplied by the estimate of volume of the region of interest $V_{\text {srCA1 }}$ to calculate the total synapse number $N_{\mathrm{SYN}}$. If the region of interest shrinks or expands during tissue preparation, without a change in the total number of synapses, the numerical density of synapses $N_{\mathrm{V}_{\mathrm{SYN}}}$ can be expected to 
increase or decrease, respectively. However, these changes in numerical density will be accompanied by a change in the volume of the region $V_{\text {srCA1 }}$ that will directly compensate for the change in numerical density $N_{\mathrm{V}_{\mathrm{SYN}}}$, so that the product of the two $\left(N_{\mathrm{V}_{\mathrm{SYN}}} \times V_{\mathrm{SrCA1}}\right)$ will be the same or the total number $N_{\mathrm{SYN}}$ will be unchanged.

One can also imagine cases of neuron loss and shrinkage, in which the numerical density remains the same, and cases of tissue expansion and neuron loss, in which the numerical density increases. The important feature of the design-based methods used here is that volume changes have no effect on the estimate of total synapse number. Measures of numerical densities by themselves are not adequate descriptors of global (within a defined region) loss or gain (Nyengaard and Gundersen 2006). Only by relating the numerical density of synapses to the volume of the structure can one make statements regarding total number and thereby loss or gain of synapses.

Changes in the dendritic arborization and in other compartments within the tissue will not have an effect on the estimates of synapse number made with the procedure used here. The six samples of numerical density in an animal, each of which was composed of eight to 12 serial electron micrographs, were placed in a systematic random manner throughout the region of interest. Samples or part of samples that included distrophic neurites, plaques (see Fig. 2), or other nonneuronal elements, such as blood vessels, were included. Because the sampling could also include plaques, vessels, and possibly distrophic dendritic processes, the number of synapses counted in each disector varied considerably. However, when the sampling is designed so that all parts of the region of interest have an equal probability of being sampled, as was done in the present study, no corrections need be made for plaques, vessels, distrophic dentrites, or vessels, in that they already have been taken into account by being included in the samples in correct proportion.

Information in the disector stacks (Table 2) can be used to evaluate the size of the objects. Numberweighted size distributions, using local estimators or serial reconstructions, can be obtained from objects that lie completely within the stack. In these cases, it is therefore of interest to have a stack that has enough sections so that the largest objects can be contained in the stack. In the example given here, the diameter of the largest, most complex synaptic contacts did not exceed $600 \mathrm{~nm}$ and the minimum cutoff for a usable serial stack was eight sections.

In the example provided here, the profiles of synaptic contacts were initially identified on each micrograph without reference to adjacent micrographs. No distinction was made among perforated, nonperforated, axospinous, and axodendritic synapses, but such a distinction has been made in several other studies (Tang et al. 2001; Ganeshina et al. 2004b). In these cases, the amount of sampling should be adjusted so that adequate samples of less numerous synaptic contact types (e.g., perforated) are obtained. Measurements of length were made across the largest axis of the individual profiles of the PSD and, in the case of perforated synapses, included the width of the perforation.

A modified stack analysis (Table 2) (Fiala and Harris 2001b; Tang et al. 2001) was used to estimate the maximum width of the synaptic contacts. The length of each of the synaptic contact profiles that belonged to the same synaptic contact was measured interactively on a computer monitor and recorded in a worksheet. The largest profile length was then determined for those synaptic contacts that were counted (i.e., had their first sectional profile below the first section in the stack) and that did not have profiles on the last, second-to-last and last, and third-to-last sections in the stack. The latter procedure ensured that contacts that were partially represented at the bottom of the stack (i.e., may not have had their maximum length within the stack) were not included in the width measures. Excluding synapses that appeared only in the bottom three sections of the stack was based on the observation that no synaptic contact could be identified in more than six consecutive sections.

The distributions of synaptic contact sizes can provide important temporal information regarding changes in synapses and thereby function changes, in that the dimensions of the contact region appear 
to be related to the functional state of the synapses. The synaptic contacts can be assigned to different size groups and standard methods used for analyzing shifts in the distributions. As a first step in this process, it is necessary to characterize the distribution. In the example provided here, the distribution has previously been characterized as a hyperbolic distribution (West et al. 2009).

\section{GLOBAL ESTIMATE OF THE TOTAL SURFACE OF SYNAPTIC CONTACTS $S_{\text {TOTAL }}$}

From the data on the length of the postsynaptic densities on each micrograph, which was used in the stack analysis, it is possible to estimate the total synaptic contact area. Using the stereological formula shown in Equation 6, which relates the length of the profiles of synaptic contacts per unit area of electron micrograph $L_{\mathrm{A}}$ to the contact surface per unit volume $S_{\mathrm{V}}$, the total contact surface $S_{\text {TOTAL }}$ was then estimated for each mouse by multiplying $S_{\mathrm{V}}$ by $V_{\mathrm{srCA} 1}$, using Equation 7. (See Estimating Surface Area in Biological Structures [West 2013d] for a more detailed discussion of surface estimators.) Information regarding the total synaptic contact area may be useful in comparative studies, although the real power of information regarding contact surface area is best obtained when one views total surface in relation to the number of synaptic contacts, that is, the mean area of a synaptic contact, or in terms of the distribution of the areas of synaptic contacts. For example, in the $\mathrm{APP}_{\mathrm{SWE}} / \mathrm{PS} 1 \Delta \mathrm{E} 9$ transgenic mouse model of Alzheimer-like amyloidosis, there is an age-related increase in the total contact area at $1 \mathrm{yr}$ of age but no increase in the mean area of individual contacts in srCA1 (West et al. 2009). In humans with Alzheimer's disease (AD), there is an increase in the mean contact area but a decrease in the total area of the contacts (Scheff et al. 2007). In the former, this indicates more synapses with no change in contact area. In the latter, it indicates fewer synapses with larger contacts.

$$
\begin{gathered}
S_{\mathrm{V}}=4 / \pi \times L_{\mathrm{A}}, \\
S_{\text {TOTAL }}=S_{\mathrm{V}} \times V_{\text {srCA }} .
\end{gathered}
$$

The variance of the estimate of $N_{\mathrm{SYN}}$, $\mathrm{VAR}_{\mathrm{N}_{\mathrm{SYN}}}$, in an individual is the sum of two variances - that for the estimate of $V_{\mathrm{REF}}$ and that for the estimate of $N_{\mathrm{V}}$ :

$$
\mathrm{VAR}_{\mathrm{N}_{\mathrm{SYN}}}=\mathrm{VAR}_{\mathrm{V}_{\mathrm{REF}}}+\mathrm{VAR}_{\mathrm{N}_{\mathrm{V}}}
$$

The variance of the estimate of $V_{\mathrm{REF}}$, that is, $\mathrm{VAR}_{\mathrm{V}_{\mathrm{REF}}}$, made with point-counting and Cavalieri techniques, can be estimated using the quadratic approximation formula (see The Precision of Estimates in Stereological Analyses [West 2012b]). The variance of the estimate of $N_{V}$, $V_{A R} R_{N_{V}}$, can be calculated with standard statistical methods for independent sampling. In this case, the number of samples is the number of stacks, six in the example given here.

The observed relative variance of each individual would be as shown in Equation 9:

$$
\mathrm{OCE}^{2} N_{\mathrm{SYN}}=\frac{\operatorname{mean}_{1-n}\left(\mathrm{VAR}_{\mathrm{V}_{\mathrm{REF}}}+\mathrm{VAR}_{\mathrm{N}_{\mathrm{V}}}\right)}{\left(\text { mean } N_{\mathrm{SYN}}\right)^{2}} .
$$

The mean observed relative variance $\overline{\mathrm{OCE}}^{2}$ is used to evaluate the efficacy of the described stereological design, as described in The Precision of Estimates in Stereological Analyses (West 2012b). The results of this analysis of the number of neurons $N$ in srCA1 in one hippocampus in each of six mice are shown graphically in Figure 6 and in Table 4. 
M.J. West

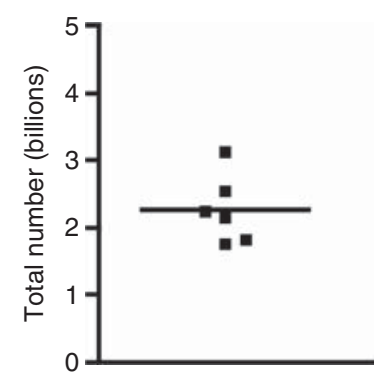

FIGURE 6. Plot of the individual estimates of total synapse number in $\operatorname{srCA} 1$ of one side of the brain in a group of six 12 -mo-old $\mathrm{C} 57 \mathrm{Bl} / 6$ mice. Horizontal bar, group mean.

\section{RATIOS OF ULTRASTRUCTURAL FEATURES: SYNAPSES PER NEURON}

The number of structural features, for example, synapse number, together with information regarding the number of cells (neurons) in the same region, can provide important insights into the structural dynamics of developmental and pathological processes. It is therefore important to ensure that cell counts be obtained from the same individual in order to take into account any covariation that might exist in the two parameters. Changes in synapse-to-neuron ratios provide a meta level of analysis of functional changes. Reduction in synapse number, without comparable reduction in neuron number, implies that a synaptotoxic process precedes neuron loss, whereas comparable changes in synapse and neuron numbers imply neuronal death as the primary process.

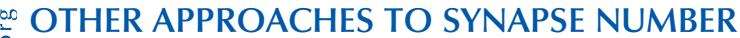

The labor-intensive, physical disector counting that is required when making estimates of synapse number from electron microscopic images raises the issue as to whether electron microscopy is absolutely necessary for the quantification of synapses. Classically, a synapse has three major components: a presynaptic element with vesicles and perimembranous specializations, a synaptic cleft, and a postsynaptic element with perimembranous specializations. The presynaptic and postsynaptic perimembranous specializations are on the inner surfaces of the cell membrane of the neurons and can be readily delineated on electron micrographs. It is generally more difficult to make a rigorous definition of the limits of the remainder of the membrane associated with a synapse. This is a problem because, as with other stereological methods, it is necessary to have rigorous structural definitions regarding when a structure is present in a section. Even in cases of synaptic boutons and dendritic spines, boundaries with the "nonsynaptic" part of the cell membrane are difficult to define. When does an axonal bouton or spine first appear in a serial stack of images when one proceeds from a dendrite to a spine? This can be expected to be a problem in a significant number of samples. The perimembranous specializations, particularly the postsynaptic specialization, however, are well defined and can be counted readily in disectors.

The perimembranous specializations of the contact region of synapses are also better indicators of the connectivity of neurons in that their size and number appear to have direct functional relationships to the amount of transmitter receptors and sites of electric current exchanges (Ganeshina et al. 2004a,b). They are, however, too small (some have diameters of fractions of a micrometer) to be

TABLE 4. Summary of data from six 12-mo-old female C57BI/6 mice

\begin{tabular}{ll}
\hline$N_{\text {SYN }} \times 10^{9}($ coefficient of variation $[C V])$ & $2.27(0.22)$ \\
$N_{\mathrm{V}_{\text {SYN }}}\left(\mu \mathrm{m}^{-3}\right)(\mathrm{CV})$ & $1.49(0.17)$ \\
$V_{\text {SrCA } 1} \times 10^{9}\left(\mu \mathrm{m}^{3}\right)(\mathrm{CV})$ & $1.52(0.09)$ \\
$S_{\text {TOTAL }} \times 10^{8}\left(\mu \mathrm{m}^{2}\right)$ & 1.24 \\
$S_{\text {Syn }}\left(\mu \mathrm{m}^{2}\right)$ & 0.055 \\
Synapse diameter $($ width $)(\mu \mathrm{m})$ & 0.301 \\
\hline$\left(N_{\text {SYN }}\right)$ Total number of synapses, $\left(N_{\left.\mathrm{V}_{\text {SYN }}\right) \text { numerical density of synapses, }\left(V_{\text {SrCA1 }}\right) \text { reference volume, }}\right.$ \\
$\left(S_{\text {TOTAL }}\right)$ total contact area, $\left(S_{\text {SYN }}\right)$ mean contact surface area.
\end{tabular}


resolved with light microscopy or confocal imaging (Fiala and Harris 2001a). Electron microscopy is necessary and, with this, the use of physical disectors. The relative speed and ease with which one can use optical disectors have prompted attempts to quantify synapses in light microscopic preparations. One approach involves the use of thick sections stained for synaptic proteins associated with presynaptic components of synapses such as synaptophysin (Calhoun et al. 1996). In these cases, one is essentially counting resolvable accumulations of a protein. Questions regarding the degree to which all synapses stain (Scheff and Price 2003) and whether adjacent synapses can be resolved temper one's enthusiasm for this approach to making global estimates of synapse number. Using counts of dendritic spines is fraught with similar difficulties. Studies of this kind are based on measures of labeled neurons with the consequence that issues of biased sampling emerge and detract from the value of the resulting data. In addition, the data, which are often presented as number per length of dendrite, are difficult if not impossible to evaluate without information regarding the total length of the dendrites.

\section{RELATED INFORMATION}

Although developments in the application of tomographic imaging to TEM (Vanhecke et al. 2007) and scanning electron microscopy (SEM) (Micheva and Smith 2007; Knott et al. 2008) have shown the potential for virtual and large-scale serial sectioning of material in which ultrastructural features can be resolved, stereological studies have yet to be performed with these methods. Regardless of how the imaging is performed, the design of stereological studies for quantifying ultrastructural features will be essentially the same as that used in the example described here.

\section{REFERENCES}

Calhoun ME, Jucker M, Martin LJ, Thinakaran G, Price DL, Mouton PR. 1996. Comparative evaluation of synaptophysin-based methods for quantification of synapses. J Neurocytol 25: 821-828.

DeGroot DMG. 1988. Comparison of methods for estimation of the thickness of ultrathin tissue sections. J Microsc 151: 23-42.

Fiala JC, Harris KM. 2001a. Cylindrical diameters method for calibrating section thickness in serial electron microscopy. J Microsc 202: 468-472.

Fiala JC, Harris KM. 2001b. Extending unbiased stereology of brain ultrastructure to three-dimensional volumes. J Am Med Inform Assoc 8: 1-16.

Ganeshina O, Berry RW, Petralia RS, Nicholson DA, Geinisman Y. 2004a. Differences in the expression of AMPA and NMDA receptors between axospinous perforated and nonperforated synapses are related to the configuration and size of postsynaptic densities. J Comp Neurol 468: $86-95$.

Ganeshina O, Berry RW, Petralia RS, Nicholson DA, Geinisman Y. 2004b. Synapses with a segmented, completely partitioned postsynaptic density express more AMPA receptors than other axospinous synaptic junctions. Neuroscience 125: 615-623.

Geinisman Y, Gundersen HJ, van der Zee E, West MJ. 1996. Unbiased stereological estimation of the total number of synapses in a brain region. J Neurocytol 25: 805-819.

Geinisman Y, Ganeshina O, Yoshida R, Berry RW, Disterhoft JF, Gallagher M. 2004. Aging, spatial learning, and total synapse number in the rat CA1 stratum radiatum. Neurobiol Aging 25: 407-416.

Hayat MA. 1979. Principles and techniques of electron microscopy: Biological applications, pp. 184-189. Van Nostrand Reinhold, New York.

Knott G, Marchman H, Wall D, Lich B. 2008. Serial section scanning electron microscopy of adult brain tissue using focused ion beam milling. J Neurosci 28: 2959-2964.

Micheva KD, Smith SJ. 2007. Array tomography: A new tool for imaging the molecular architecture and ultrastructure of neural circuits. Neuron 55: 25-36.

Nyengaard JR, Gundersen HJ. 2006. Direct and efficient stereological estimation of total cell quantities using electron microscopy. J Microsc 222: 182-187.
Scheff SW, Price DA. 2003. Synaptic pathology in Alzheimer's disease: A review of ultrastructural studies. Neurobiol Aging 24: 1029-1046.

Scheff SW, Price DA, Schmitt FA, DeKosky ST, Mufson EJ. 2007. Synaptic alterations in CA1 in mild Alzheimer disease and mild cognitive impairment. Neurology 68: 1501-1508.

Tang Y, Nyengaard JR, De Groot DM, Gundersen HJ. 2001. Total regional and global number of synapses in the human brain neocortex. Synapse 41: $258-273$.

Vanhecke D, Studer D, Ochs M. 2007. Stereology meets electron tomography: Towards quantitative 3D electron microscopy. J Struct Biol 159: 443-450.

West MJ. 2012a. Estimating volume in biological structures. Cold Spring Harb Protoc doi: 10.1101/pdb.top071787.

West MJ. 2012b. The precision of estimates in stereological analyses. Cold Spring Harb Protoc doi: 10.1101/pdb.top071050.

West MJ. 2012c. Systematic versus random sampling in stereological studies. Cold Spring Harb Protoc doi: 10.1101/pdb.top071837.

West MJ. 2012d. Estimating object number in biological structures. Cold Spring Harb Protoc doi: 10.1101/pdb.top071423.

West MJ. 2013a. Optimizing the sampling scheme for a stereological study: How many individuals, sections and probes should be used. Cold Spring Harb Protoc doi: 10.1101/pdb.top071852.

West MJ. 2013b. Getting started in stereology. Cold Spring Harb Protoc doi: 10.1101/pdb.top071845.

West MJ. 2013c. Isotropy, iSectors, and vertical sections in stereology. Cold Spring Harb Protoc doi: 10.1101/pdb.top071803.

West MJ. 2013d. Estimating surface area in biological structures. Cold Spring Harb Protoc doi: 10.1101/pdb.top071829.

West MJ, Bach G, Søderman A, Jensen JL. 2009. Synaptic contact number and size in stratum radiatum CA1 of $\mathrm{APP} / \mathrm{PS} 1 \Delta \mathrm{E} 9$ transgenic mice. Neurobiol Aging 30: 1756-1776.

Witgen BM, Grady MS, Nyengaard JR, Gundersen HJ. 2006. A new fractionator principle with varying sampling fractions: Exemplified by estimation of synapse number using electron microscopy. J Microsc 222: 251-255. 


\section{Counting and Measuring Ultrastructural Features of Biological Samples}

Mark J. West

Cold Spring Harb Protoc; doi: 10.1101/pdb.top071886

\begin{tabular}{rc}
$\begin{array}{r}\text { Email Alerting } \\
\text { Service }\end{array}$ & Receive free email alerts when new articles cite this article - click here. \\
\hline $\begin{array}{c}\text { Subject } \\
\text { Categories }\end{array}$ & $\begin{array}{c}\text { Browse articles on similar topics from Cold Spring Harbor Protocols. } \\
\text { Cell Biology, general (1382 articles) }\end{array}$ \\
& $\begin{array}{l}\text { Electron Microscopy (43 articles) } \\
\text { Image Analysis (124 articles) } \\
\text { Neuroscience, general (357 articles) }\end{array}$ \\
\hline
\end{tabular}

